109

INFANT TEMPERAMENT ACCORDING TO RISK STATUS ANO TYPE OF DELIVERY C. J. R. Simons, Martha K. Mullett, Jones) WVU School of Medicine, Department of Pediatrics, Morgantown, WV 26506

The present study examined both parents' ratings of infant temperament according to the infant's risk status and type of temperament according to the infant's risk status and type of
delivery. Ninety parents rated their 45 infants, 23 of whom were low-risk and 22 who were high-risk with medical complications, on the Revised Infant Temperament Questionnaire (RITQ). Type of delivery was not related to the easy-difficult ratings of the high-risk babies; however, a strong trend $(p<.067)$ emerged in the low-risk sample with $C$-section babies receiving more difficult ratings than the vaginally-delivered infants. As the easy-difficult ratings of the RITQ are determined by 5 individual profiles, these profiles were analyzed according to risk status and delivery type. The high-risk infants received more positive ratings on the mood profile than the low-risk infants $(p<.04)$, while low-risk, C-section babies received the most negative ratings on mood $(\mathrm{p}<.04)$. Low-risk, C-section and high-risk, vaginally-delivered infants were rated more withdrawing than the low-risk, vaginally-delivered and high-risk, $c$-section babies $(p<.001)$. In addition, there were trends for the high-risk babies to receive more rhythmic ratings than the low-risk infants $(p<.06)$; vaginally-delivered infants were rated more adaptable than the $\mathrm{C}$-section babies $(\mathrm{p}<.053)$; and low-risk, C-section babies were rated the least adaptable of al1 the infants $(p<.07)$.

THE IMPACT OF NEONATAL NUKSE CLINICIANS AS PRIMARY

110 PROVIDERS ON MORBIDITY AND MORTALITY OF VERY LOW BIRTH WEIGHT PREMATURE INFANTS. Maureen Sims, Naveen Jasani, Jean Yan, Joan Hodgman. USC Sch of Med., LAC-USC ed. Ctr., Dept. of Peds. and Nursing, Los Angeles.

Many medical facilities have introduced non-physicians as primary care providers for high risk neonates. Our medical center developed a neonatal nurse clinician (NNC) program in response to the reduction of numbers of pediatric housestaff the perceived importance of consistent primary care providers, and the need for a career ladder for direct patient care in nursing. The object of this study was to evaluate the influence of NNCs on mortality and morbidity using peri and intraventricular hemorrhage (PV/IVH) as a marker for infants with BW 750-1250g. 42 successive inborn infants $(B W=1038 \pm 148 \mathrm{~g}, G A=29 \pm 2$ wks) who received primary care from pediatric housestaff were prospectively evaluated for PV/IVH and survival in 1981. In 198274 infants ( $B W=1010 \pm 154 \mathrm{~g}, G A=28 \pm 2$ wks) received primary care from NNCs and were evaluated for PV/IVH and survival. Primary care included: resuscitation; maternal history; physical exams; procedures ( $i$. e., intubation, thoracentesis, chest tube insertion, and umbilical catheterization), clinical assessment and management plans. Results:

Survival Without PV/IVI 42 (268) 74

Total Survival PV/IVH 11 (268) $37 / 65 t(578)<.01$

Conclusion: The NNC has contributed to reducing morbidity and mortality for VLBW premature infants. ( $\dagger 9$ infants not scanned for PV/IVH.

\section{LATE APPEARANCE OF EMOTIONAL DISTURBANCE AS A}

111 SEQUELA OF PREMATURITY. Richard $H$. Smith, I. Mark St. Peter's Medical Center, Dept. of Pediatrics N ew Bumsick, N.J. Emotional disturbance associated with prematurity may not appear until the adolescent years. Referral for special ed. on
the basis of substantial classroom difficulty is a documented complication of prematurity. A representative sample of 500 Emotionally Disturbed Special Education classified students had at least $12 \%$ preterm birth rate with $4 \%$ weighing below $2000 \mathrm{~g}$ at birth. The emotional disorder frequently began after age $12 y r s$. as a disturbance in conduct. The child's behavior was due to frustration in reaction to learning disability and to overproction by parents concerned about the ir child's vuinerability. The psychiatric diagnosis and academic achievement of classified str dents with preterm births differed from classified students with term births, but the age of onset was similar. Special Ed. for Emotionally Disturbed classification contrasts with other classifications in New Jersey in the age of onset:

\begin{tabular}{|c|c|c|}
\hline$\frac{\text { Classification }}{\text { Orthopedic handicap }}$ & $\frac{75 \% \text { classified by }}{5 \text { years of age }}$ & $\frac{95 \% \text { classified by }}{7 \text { years of age }}$ \\
\hline Communication handicap & $-"-$ & $-"-$ \\
\hline Neurological impairment & 7 & $-"$ \\
\hline Perceptual impairment & - & 11 \\
\hline otional dis & 12 & 15 \\
\hline
\end{tabular}

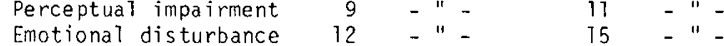

Since emotional disturbance may present in adolescent yrs., follow up of greater than 10 years is necessary to evaluate the incidence of emotional disturbance in infants with preterm births. PHENOBARBITAL IMPROVES MOTOR MATURITY IN PREMATURE
112 InFANTS. Richard $H$. Smith, Barbara M. Ostfeld, Mujahid Anwar, I. Mark Hiatt, Thomas Hegyi. UMDNJRutgers Medical School, St. Peter's Medical Center, Department of Pediatrics, New Brunswick, N. J.

$\mathrm{Higher}$ scores on motor maturity items of the Brazelton Neonatal Behavioral Assessment Scale (BNBAS) were observed in a group of infants treated with phenobarbital $(P)$. Twenty three infants ranging in $\mathrm{BW}$ from 900 to $1480 \mathrm{~g}$ were randomly assigned to treatment $(N=9)$ or control $(N=14)$ groups in a trial evaluating the efficiency of $P$ in preventing intraventricular hemorrhage (IVH). Study infants were treated for 7 days with anticonvulsant doses achieving $P$ levels of $20-30 \mathrm{ug} / \mathrm{ml}$.

The BNBAS was performed a week prior to discharge at a mean post-conceptual age of $37+2 w k s$. The two groups were comparable with respect to incidence of IVH, age at evaluation, and number of abnormal neurologic responses assessed by the BNBAS. Treated infants scored higher in motor maturity $(P<0.001)$ while controls had greater tremulousness $(P<0.001)$. Cuddliness was higher $(P<0.05)$ in the treated group, who were more mature in behavioral responses than controls. Comparing 7 study and 13 control infants with Grade III IVH, we noted auditory responsiveness, pull to sit, motor maturity, and hand to mouth facility to be significantly better in the treated group $(P<0.05)$.
113 ATTENTION DEFICIT DISORDER: IMPLICATIONS FOR MECHAN ISMS OF STIMULANT DRUG ACTION. Mary $V$. Solanto(Spon by M.I.Cohen), Albert Einstein College of Medicine, N.Y., Dept. of Pediatrics.

The mechanism of therapeutic action of stimulants in children with Attention Deficit Disorder (ADD) is poorly understood. In animals, stimulation of post-synaptic dopamine receptors by $\mathrm{d}$ amphetamine causes an increase in locomotor activity at low doses, but a decrease in activity with increased stereotypy at higher doses. Clinical and experimental reports suggest that the decrease in activity and increase in focused attention in children following stimulants are analogous to stimulant effects seen in animals at higher doses; if so, one would predict an increase in activity at sub-clinical doses. Twelve children meeting DSM III criteria for ADD were evaluated for activity (quadrant changes and toy changes in a playroom) and attentiveness (Children's Checking Task) following placebo and following a sub-clinical dose of methylphenidate $(.1 \mathrm{mg} / \mathrm{kg})$ in a double-blind crossover design. Rather than increasing activity as predicted, methylphenidate decreased by half the mean numbers of quadrant changes and toy changes $(p<.05$ by 2 -tailed $t$-test). Interestingly, there were no drug effects on attention. The results suggested that stimulant drugs may decrease activity by stimulating inhibitory autoreceptors on pre-synaptic dopamine neurons. Such effects have been demonstrated in animals following d-amphetamine at doses which are lower than those which stimulate postsynaptic receptors, and which are compatible with dosages used clinically.

IN PRETERM

114 INFANTS. Mark Stefanski, Kar1 Schulze, Ju1ia Columbia Univ.,Col1.P \&S,Babies Hosp.,Div. Perin., Dept. Ped., N.Y. This study was undertaken to quantitate the $24 \mathrm{hr}$. variability in the distribution of states for 5 healthy preterm infants. 12 studies were conducted in which state was coded by independent EEG \& behavioral scores for each minute under standard nursing conditions over a continuous $24 \mathrm{hr}$. period excluding feeding times ( $\bar{x}$ study time $=1225$ mins). We calculated the percent time Quiet Sleep (\%QS), Active Sleep (\%AS), Indeterminate Sleep (\%IS), and Wakefulness (\%) for 3 post-conceptional age groups:

$\begin{array}{lllllr} & & \% Q S & \% \text { AS } & \% \text { IS } & \% W \\ >34 \text { wks. } & (n=6) & 12.1 & 75.1 & 8.9 & 3.7 \\ >34 \text { \& <37 wks. } & (n=3) & 13.3 & 73.9 & 6.5 & 6.2 \\ \geq 37 \text { wks. } & (n=3) & 18.0 & 65.2 & 5.4 & 11.4\end{array}$

These data demonstrate increased $W$ and better organization of sleep with maturation. State distribution was also calculated for each interfeed epoch $(n=8)$ to obtain standard deviations (SD) for $\% Q S, \% A S, \% I S \& \% \mathrm{~W}$ in each study. The average SD ( $\bar{x} S D)$ for each state was used to determine the variability in estimates of $24 \mathrm{hr}$. state distribution when less than 8 epochs are observed: $\begin{array}{cccccccccc} & \overline{\mathrm{X}} & 1 & 2 & 3 & 4 & 5 & 6 & 7 & 8 \\ \% \text { QS } & 13.9 & 6.45 & 4.56 & 3.72 & 3.22 & 2.88 & 2.63 & 2.43 & 2.28\end{array}$ $\begin{array}{llllllllll}\% \text { AS } & 70.9 & 8.92 & 6.30 & 5.14 & 4.46 & 3.98 & 3.64 & 3.37 & 3.15\end{array}$ $\bar{X} S D$ for $\% Q S$ when only 1 interfeed epoch is used is 6.45 ; when 8 epochs are used $\overline{\mathrm{X}} \mathrm{SD}=2.28$. The results demonstrate the importance of long term measurements of state distribution. Short term observations may be misleading. 